\title{
Bronchiolitis obliterans organising pneumonia associated with the use of nitrofurantoin
}

\author{
R J Cameron, J Kolbe, M L Wilsher, N Lambie
}

\begin{abstract}
The spectrum of nitrofurantoin lung injury continues to widen. The case histories are presented of two patients who developed lung disease associated with the use of nitrofurantoin with histological features of bronchiolitis obliterans organising pneumonia (BOOP), a rare but recognised form of drug induced injury. The two middle aged women presented with respiratory symptoms after prolonged treatment with nitrofurantoin. Both had impaired lung function and abnormal computed tomographic scans, and their condition improved when nitrofurantoin was withdrawn and corticosteroid treatment commenced. The favourable outcome in these two patients contrasts with the fatal outcome of the two other reported cases of nitrofurantoin induced BOOP. We suggest that the previous classification of nitrofurantoin induced lung injury into "acute" and "chronic" injury is an oversimplification in view of the wide variety of pathological entities that have subsequently emerged. (Thorax 2000;55:249-251)
\end{abstract}

Keywords: bronchiolitis obliterans organising pneumonitis; drug induced pulmonary disease; nitrofurantoin

An increasing number of drugs are recognised as causing lung injury and the spectrum of their adverse effects is widening. A recognised but uncommon form of drug induced lung disease is bronchiolitis obliterans organising pneumonia (BOOP). ${ }^{1}$ We report two cases of nitrofurantoin induced pulmonary disease with histological features of BOOP.

Respiratory Services, Green Lane Hospital, Green Lane West, Auckland 3, New

Zealand

R J Cameron

J Kolbe

M L Wilsher

Department of Pathology

N Lambie

Correspondence to: Dr J Kolbe

Received 25 November 1997 Returned to authors 19 January 1998

Revised manuscript received

2 June 1998

Accepted for publication

10 July 1998 thickening, especially of the medium and small sized bronchi, with very little fibrosis. Open lung biopsy specimens showed that many respiratory bronchioles were distorted and largely occluded by fibroblastic tissue with associated mucus plugging and outgrowth of respiratory epithelium into surrounding alveolar tissue, consistent with BOOP. In the absence of other factors a diagnosis of nitrofurantoin induced pulmonary disease was made and the drug was discontinued. Prednisone $30 \mathrm{mg}$ per day, gradually reducing over nine months, resulted in significant symptomatic improvement, significant improvement in lung function $\left(\mathrm{FEV}_{1} 3.56 \mathrm{l}\right.$, FVC 4.201 , TLCO $82 \%$ predicted), and considerable but incomplete clearance of interstitial changes on the HRCT scan.

\section{Case 2}

A 50 year old female non-smoker with recurrent urinary tract infections gave a two month history of worsening dyspnoea, fatigue, anorexia, and cough with fevers and night sweats for three weeks. There was no history to suggest an underlying connective tissue disorder. She had been taking nitrofurantoin $50 \mathrm{mg}$ at night regularly for one year. On examination she was tachypnoeic and tachycardic with bi-basal "velcro" crackles. Arterial blood gas measurements showed hypoxia $\left(\mathrm{PaO}_{2} 6.5 \mathrm{kPa}\right)$ with a wide alveolar-arterial gradient $(9.1 \mathrm{kPa})$. Blood count and renal and liver function were normal, erythrocyte sedimentation rate (ESR) was $81 \mathrm{~mm} / \mathrm{h}$, and the ANA was 1:1640 with anti dsDNA negative. Lung function tests showed $\mathrm{FEV}_{1}$ of 0.821 and FVC of 0.841 (predicted 2.87 and 3.771 , respectively). TLCO could not be measured because of breathlessness. An HRCT scan of the thorax showed patches of "ground glass" opacity, interstitial fibrosis with traction bronchiectasis, and scattered areas of dense consolidation (fig 1). Transbronchial biopsy specimens showed loose immature fibrous tissue within air spaces and incorporated into the interstitium, a patchy interstitial infiltrate of mixed inflammatory cells including lymphocytes, plasma cells, and a few eosinophils, and prominent hyperplasia of type II pneumocytes. Pieces of airway wall showed inflammation with peri-airway fibrous and outgrowth of respiratory epithelium into fibrotic lung tissue. The appearance was consistent with BOOP/diffuse alveolar damage. The diagnosis of nitrofurantoin induced pulmonary disease was made; the drug was withdrawn and prednisone $40 \mathrm{mg}$ daily reducing slowly to baseline $10 \mathrm{mg}$ daily was given over three months. At three months a repeat 

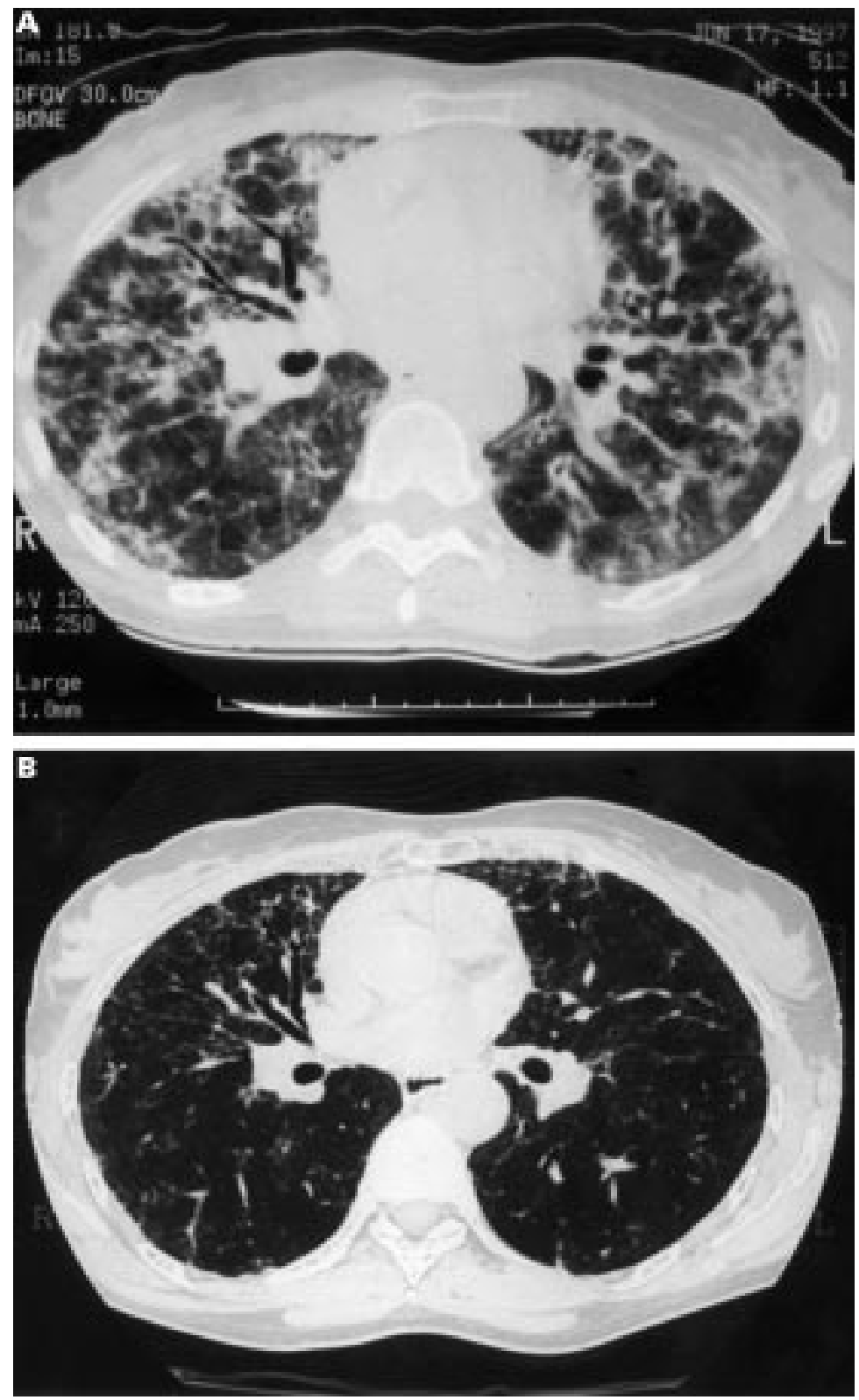

Figure 1 (A) Mid thoracic HRCT scan in case 2 showing small residual areas of normal lung, extensive interstitial fibrosis with traction bronchiectasis most marked in the right middle lobe, patchy ground glass opacity, and areas of dense consolidation. (B) Equivalent HRCT scan three months after withdrawal of nitrofurantoin and commencement of treatment with prednisone showing extensive but incomplete clearance of abnormalities.

HRCT scan showed marked reduction of the ground glass opacities and areas of consolidation, but with persistent interstitial fibrosis. Repeat lung function tests showed $\mathrm{FEV}_{1}$ had improved to 2.881 (100\% predicted) with FVC 2.891 ( $77 \%$ predicted) and TLCO $66 \%$ predicted. The patient was subsequently weaned off oral steroids with no clinical, radiological, or physiological evidence of relapse.

\section{Discussion}

We conclude that both patients had nitrofurantoin induced pulmonary disease on the grounds that there was a lack of an alternative explanation for their lung disease and a good response to drug withdrawal and treatment with an oral corticosteroid. We acknowledge that BOOP of other causes may respond well to corticosteroid treatment, but there was no disease recrudescence on steroid reduction and withdrawal. The establishment of a firm aetiological relationship would require re-challenge with nitrofurantoin. This was considered inappropriate in view of the severity of pulmonary impairment on presentation and the residual and irreversible changes on the HRCT scan.

Relatively few pharmaceutical agents have been associated with BOOP. These include amiodarone, acebutalol, nilutamide, cephalosporins, barbiturates, and cocaine. ${ }^{2}$ There are only two previously reported cases of BOOP attributable to nitrofurantoin use. ${ }^{3}$ Both patients were elderly ex-smokers with symptoms of 3-4 weeks duration and both responded well to initial corticosteroid treatment, but rapid tapering led to an irreversible decline and death after failure to respond to increased steroid dosage. Details of drug treatment were not included in the report.

The course of the disease in our patients was rather different. Both were maintained on medium to high dose prednisone initially, gradually reducing over months, and the duration of treatment may have been important in terms of the improved outcome. They were weaned off oral steroids without clinical, radiological, or physiological evidence of relapse.

Nitrofurantoin induced pulmonary disease may present in many forms including BOOP, diffuse alveolar damage, vasculitis, interstitial fibrosis, pleural and airways disease, and pulmonary haemorrhage. ${ }^{1}$ A final common toxic pathway has not been postulated. Nitrofurantoin induced pulmonary disease may result from immune mediated injury ${ }^{3}$ or via hydroxyl radical generation with subsequent free oxidant damage. ${ }^{5}$ The reduced incidence with the addition of the antioxidant ascorbic acid to nitrofurantoin preparations ${ }^{5}$ and results of in vitro studies ${ }^{6}$ suggest that this and other antioxidants may significantly reduce toxicity.

Initial reports suggested that the duration of nitrofurantoin treatment dictated the disease pattern. The "acute" reaction was characterised by marked constitutional symptoms including rash, fever, arthralgia, fatigue, together with pulmonary symptoms of dry cough and dyspnoea..$^{3-5}$ The "subacute" and "chronic" forms were more insidious, with increased eosinophil count, raised ESR, and vasculitis and interstitial inflammation on histological examination, consistent with a type III immune response. ${ }^{3}$ Increased immunoglobulin levels, hepatic transaminases and ANA titres (the so called "drug induced lupus syndrome"7 8 ) was associated with a degree of irreversible fibrosis. Some early reports of biopsy specimens from a patient with nitrofurantoin induced lung disease ${ }^{5}$ which predate the recognition of idiopathic BOOP as an independent entity are suggestive of a BOOP-like pattern. Cohen ${ }^{3}$ suggested that BOOP may be a precursor to chronic lung fibrosis, an early and potentially reversible phase in the spectrum of fibrosing lung disease. However, both patients in this report had residual radiological abnormalities although the remaining functional abnormalities were minor. The subsequent variety of 
pathological entities now shown to be caused by nitrofurantoin suggests that these early categorisations are an oversimplification.

The initial interest in nitrofurantoin induced lung disease has waned as more suitable less toxic agents have been found for chronic urinary infections. However, the drug remains generally available in spite of its high toxic profile and clinicians need to be aware of the spectrum of associated lung disease.

1 Fourcher P, Biour M, Blayac JP, et al. Drugs that may injure the respiratory system. Eur Respir f 1997;10:265-79.
2 Cordier J-F. Cryptogenic bronchiolitis obliterans organising pneumonia Chin Chest Med 1993;14:677-92.

3 Cohen AJ, King TE Jr, Downey GP. Rapidly progressive bronchiolitis obliterans with organising pneumonia. $A m \mathcal{F}$ Respir Crit Care Med 1994;149:1470-5

4 Holmberg L, Boman G, Bottinger LE, et al. Adverse reactions to nitrofurantoin: analysis of 921 reports. Am J Med 1980;69:733-8.

5 Sovijarvi ARA, Lemola M, Stenius B, et al. Nitrofurantoininduced acute, subacute and chronic pulmonary reactions. A report of 66 cases. Scand F Respir Dis 1977;58:41-50.

6 Martin WJ II. Nitrofurantoin: evidence for oxidant injury of lung parenchymal cells. Am Rev Respir Dis 1983;127:482-6.

7 Hailey FJ, Glascock HW, Hewitt WF. Pleuropneumonic reactions to nitrofurantoin. $\mathrm{N} \mathrm{Engl} \mathrm{F} \mathrm{Med} \mathrm{1969;281:1087-90.}$

8 Bäck O, Lundgren R, Wiman LR. Nitrofurantoin-induced pulmonary fibrosis and lupus syndrome. Lancet 1974;i:
930 .

\section{LETTERS TO THE EDITOR}

\section{Systematic review of antistaphylococcal antibiotic therapy in cystic fibrosis}

McCaffery et al" conclude that "antistaphylococcal treatment achieves sputum clearance of Staphylococcus aureus in patients with cystic fibrosis ... ." and that prophylactic treatment in young children is ". . . likely to be of clinical benefit". These positive conclusions are based on the results of a study which has important methodological problems. Neither the introduction nor the methods section of this review state what hypotheses the review set out to test, the criteria used to decide whether a study was suitable for inclusion, outcomes to be studied in the review, or methods used to assess the methodological quality of included studies. Systematic reviews differ from narrative reviews in that they test hypotheses using a methodology which is well described. ${ }^{2}$ The authors have described their search strategy, which is based on that developed by the Cochrane Collaboration, to identify randomised controlled trials. The authors have, however, included a number of studies in their review which are not randomised controlled trials. It is not clear from the information provided whether their search strategy is sensitive enough to identify all possible relevant studies.

The authors base their conclusions on the results of just two randomised controlled trials, involving only 66 children, with a maximum follow up of two years. ${ }^{3}$ All of these children were under seven years of age (most under two years) and had upper respiratory samples taken, not sputum. Of the other studies described as randomised, one used alternate allocation (and so was not randomised) ${ }^{5}$ and one reported further outcomes in patients included in one of the randomised controlled trials. ${ }^{6}$ Only two randomised controlled trials actually reported the prevalence of $S$ aureus in respiratory secretions. The larger study by Weaver $e t a l^{\beta}$ reported that the prevalence of $S$ aureus was reduced with prophylaxis but "clearance" was not achieved from nose and throat swabs.

The important issues for cystic fibrosis patients and their families are not eradication of an organism but fewer symptoms, improved lung function, and prolonged survival. None of the studies described in the review addressed these issues nor, indeed, the concern that prophylactic antibiotics may encourage chronic pulmonary infection with organisms such as Pseudomonas aeruginosa or multiply resistant $S$ aureus. A Cochrane review addressing this intervention is currently being undertaken by two of us (AS and SW) and this will have the advantage that it will be regularly updated to incorporate new studies. Only when further properly designed studies have been completed and included in an up to date systematic review will we be able to evaluate whether antistaphylococcal antibiotic prophylaxis is ". . . likely to be of clinical benefit”.

ALAN SMYTH

Children's Cystic Fibrosis Unit, Nottingham City Hospital, Nottingham NG5 1PB, UK

SARAH WALTERS

Department of Public Health and Epidemiology, University of Birmingham, Birmingham B15 2TT, UK ROSALIND SMYTH

University of Liverpool Department of Child Health, Roval Liverpool Children's Hospital, Liverpool L12 2AP, UK

1 McCaffery K, Olver RE, Franklin M, et al. Systematic review of antistaphylococcal antibiotic therapy in cystic fibrosis. Thorax 1999;54:3803.

2 Clarke M, Fischer M, Moustgaard R, et al. The Cochrane Collaboration Handbook Version 3.0.2. Cochrane Library Issue 3. Oxford: Update Software, 1999.

3 Weaver LT, Green MR, Nicholson K, et al. Prognosis in cystic fibrosis treated with continuous flucloxacillin from the neonatal period. Arch Dis Child 1994;70:84-9.

4 Schlesinger E Muller W, von der Hardt $\mathrm{H}$, et al. Continuous antistaphylococcal antibiotic treatContinuous antistaphylococcal antibiotic treatment in young children with cystic fibrosis. 9 th International Cystic Fibrosis Congress 1984;
4.14(abstract). 4.14(abstract)

Harrison CJ, Marks MI, Welch DF. A multicentric comparison of related pharmacologic features of cephalexin and dicloxacillin given for two months to young children with cystic fibrosis. Pediatr Pharmacol 1985;5:7-16.

6 Beardsmore CS, Thompson JR, Williams A, et al. Pulmonary function in infants with cystic fibrosis: the effect of antibiotic treatment. Arch Dis Child 1994;71:133-7.

AUTHORS' REPLY Smyth et al have listed a number of features that they regard as being essential to systematic reviews. In particular, they suggest that systematic reviews should always test hypotheses. However, a clear understanding of the existing evidence is necessary for the generation of valid hypo- theses and, in our view, this is best achieved by systematic reviewing. Indeed, many important systematic reviews published in major clinical journals do not specifically test hypotheses, but study the current evidence in order to identify the state of existing knowledge and to define areas for further research ${ }^{12}$ This objective is consistent with the view of the authors of the Cochrane Collaboration Handbook who recognise that systematic reviews can have different motivations, one of which is the resolution of conflicting evidence. ${ }^{3}$ Indeed, it is probably difficult to define systematic reviews as formally as Smyth et al (and others) have proposed as the science of systematic reviewing is undergoing continuous development. More systematic reviews are being performed now than ever before (a Medline search looking for "systematic review" in titles and abstracts presents 4158 citations in the last 10 years, $1538(37 \%)$ of which are in the last two), with reviewers defining their methods according to the problem in question.

Again, because of the nature of the field being studied, we had purposely not defined stricter criteria for study selection or drawn up a preselected list of outcomes of interest. As the area under investigation was largely unknown, we felt such criteria could limit our search. Also, in the absence of any significant background information, we were uncertain if such a choice of outcomes could be made objectively. Indeed, if we had arbitrarily drawn up a list of outcomes that were of interest to us, we would have missed a number of outcomes that others had used and which could be of potential interest to readers when designing clinical trials in the future. We did not use quality scores because there is little objective evidence to support the use of quality scoring in systematic reviews. ${ }^{4}$ Many of the scoring systems have not been developed with sufficient rigour ${ }^{4}$ and could add the analyst's bias to the results. ${ }^{5} \mathrm{~A}$ recent review of a random sample of 240 metaanalyses showed that less than half assessed trial quality. ${ }^{6}$ However, we note that newer techniques such as meta-regression may provide better alternatives in the future.

As we were principally interested in randomised controlled trials (RCTs), we used a search strategy that has been well validated for the recall of such trials. However, as before, ${ }^{7}$ we wanted to present an analysis of outcomes of both RCTs and non-RCTs because we felt this would make our conclusions more objective. Again, this approach is supported by the authors of the Cochrane Collaboration Handbook. ${ }^{3}$ Smyth et al state quite rightly that the important issues for cystic fibrosis patients and their families are not eradication of an organism but fewer 
symptoms, improved lung function, and prolonged survival. However, this should not inhibit the use of laboratory based outcomes which could influence clinical decision making until appropriate clinical data are available. Indeed, given the high predictive value of oropharyngeal cultures in children for identifying pathogens in bronchoalveolar lavage fluid (sensitivity and specificity of $90 \%$ ), we feel the evidence we have defined in support of clearance of Staphylococcus aureus from the upper or lower respiratory tract with anti-staphylococcal antibiotics does suggest that this therapeutic intervention is likely to be of clinical benefit, although we strongly support their argument that properly designed studies are needed to confirm this hypothesis.

\section{KEVIN MCCAFFERY RICHARD E OLVER MARGARET FRANKLIN SOMNATH MUKHOPADHYAY \\ Centre for Research into Human Development, Ninewells Hospital $\mathcal{G}$ Medical School, Dundee \\ DD1 9SY, UK}

1 Campbell NC, Ritchie LD, Cassidy J, et al. Systematic review of cancer treatment pro-
grammes in remote and rural areas. Brf $\mathrm{Cancer}$ 1999;80:1275-80.

2 Lipworth BJ. Systemic adverse effects of inhaled corticosteroid therapy: a systematic review and meta-analysis. Arch Intern Med 1999;159:94155 .

3 Mulrow CD, Oxman AD. The Cochrane Collaboration Handbook Version 3.0.2. Cochrane Li-

4 Moher D, Jadad AR, Tugwell P. Assessing the quality of randomized controlled trials. Current issues and future directions. Int $\mathcal{F}$ Techno Assessment Health Care 1996;12:195-208.

5 Greenland S. Invited commentary: a critical look at some popular meta-analytic methods. Am f Epidemiol 1994;140:290-6.

6 Moher D, Cook D, Jadad A, et al. Assessing the quality of reports of randomised trials: implications for the conduct of meta-analyses. Health Technol Assessment 1999;3:1-98.

7 Mukhopadhyay S, Singh M, Cater JI, et al. Nebulised anti-pseudomonal antibiotic therapy in
cystic fibrosis: a meta-analysis of benefits and cystic fibrosis: a meta-analysis
risks. Thorax 1996;51:364-8.

8 Avital A, Uwyyed K, Picard E, et al. Sensitivity and specificity of oropharyngeal suction versus bronchoalveolar lavage in identifying respiratory tract pathogens in children with chronic pulmonary infection. Pediatr Pulmonol 1995;20:

\section{Therapeutic ratio of inhaled fluticasone}

I read with interest the recent article by Meijer and colleagues on the effects of inhaled fluticasone and prednisolone on clinical and inflammatory parameters in patients with asthma. ${ }^{1}$ Rather than focusing on the differences between oral and inhaled corticosteroid, I believe that a more important finding is the effect of a fourfold increase in the dose of fluticasone on the therapeutic ratio. For airway parameters there were no significant differences in the effects on bronchial hyperresponsiveness to methacholine and adenosine monophosphate or on sputum eosinophils between fluticasone in doses of $500 \mu \mathrm{g}$ and $2000 \mu \mathrm{g}$ per day. However, for systemic bioactivity markers there were significant differences between the two doses of fluticasone on serum cortisol levels and blood eosinophils. Taken together these findings suggest that, at least for effects on airway hyperresponsiveness and inflammation, the therapeutic ratio for fluticasone declines sharply above a watershed dose of $500 \mu \mathrm{g}$ per day. This result is perhaps not surprising, given the high glucocorticoid topical potency for in vitro anti-inflammatory activity with fluticasone. ${ }^{2}$

It is also important to point out that the study by Meijer et al was performed using fluticasone delivered via a Diskhaler dry powder inhaler device, which delivers a twofold lower respirable fine particle dose than a fluticasone propionate pressurised metered dose inhaler. ${ }^{3}$ This is due to the larger particle size from the fluticasone dry powder inhaler. Hence, increasing the nominal dose of fluticasone dry powder may result in a proportionately greater delivery of larger particles to the central airways and consequently to a less than expected impact on small airway inflammation. The lower fine particle dose of fluticasone dry powder will also result in reduced lung bioavailability, as shown by a fivefold lower degree of adrenal suppression compared with the same nominal dose of fluticasone delivered via a pressurised metered dose inhaler with spacer device. ${ }^{4}$ The use of fluticasone in a dose of $500 \mu \mathrm{g} /$ day via a dry powder inhaler would therefore explain the absence of any significant suppression of blood eosinophils or serum cortisol in their study. This does not mean that fluticasone propionate dry powder in a dose of $500 \mu \mathrm{g} /$ day is not systemically bioavailable, as recently published data with this dose of fluticasone given via a Diskhaler reported significant suppression of 24 hour urinary cortisol excretion (33\% reduction) and peripheral blood lymphocyte glucocorticoid receptor mRNA expression ( $71 \%$ reduction) during steady state dosing in asthmatic patients.

Another finding in the study by Meijer et al was the relatively greater effect on bronchial hyperresponsiveness to adenosine monophosphate than to methacholine challenge with both oral and inhaled corticosteroid after two weeks. Similar findings have been reported after two weeks of treatment with inhaled budesonide powder in a dose of $1600 \mu \mathrm{g} /$ day. ${ }^{6}$ The authors not unreasonably suggested that adenosine monophosphate responsiveness might be more sensitive to changes in airway inflammation than methacholine. However, the treatment period was relatively short and one cannot exclude the possibility that the effects on methacholine hyperresponsiveness might have been proportionately greater with a longer duration of treatment, as has been reported in previous studies. ${ }^{78}$ It is also conceivable that differences in bronchial hyperresponsiveness between the doses of inhaled fluticasone may have become apparent with a longer duration of treatment.

Finally, it is important not to extrapolate the results of the study by Meijer et al on patients with relatively mild asthma to more severe asthmatic patients in whom altered airway geometry may cause a reduction in lung delivery and lung bioavailability from narrowed peripheral small airways. Also, their results may be specific to the unique drug/device interaction of fluticasone propionate given via the dry powder inhaler, and further studies are needed to look at the dose-response relationship for the therapeutic ratio using more efficient delivery systems such as a pressurised metered dose inhaler with spacer.

BRIAN J LIPWORTH Asthma and Allergy Research Group, Department of Clinical Pharmacology, Ninewells Hospital and Medical School, University of Dundee, Dundee DD1 9SY,
1 Meijer RJ, Kerstiens HAM, Arends LR, et al. Effects of inhaled fluticasone and oral prednisolone on clinical and inflammatory parameters in patients with asthma. Thorax 1999;54:894-9.

2 Stellato C, Atsuta J, Bickel CA, et al. An in-vitro comparison of commonly used topical glucocorticoid preparations. F Allergy Clin Immunol 1999;104:623-9.

3 Olsson B. Aerosol particle generation from drypowder inhalers: can they equal pressurized metered dose inhalers? 7 Aerosol Med 1995;8: S13-9.

4 Wilson AM, Dempsey OJ, Coutie WJ, et al. Importance of drug-device interaction in determining systemic effects of inhaled corticomining systemic effects of inh

5 Andersson O, Cassel TM, Gronneberg R, et al. In vivo modulation of glucocorticoid receptor mRNA by inhaled fluticasone propionate in bronchial mucosa and blood lymphocytes in subjects with mild asthma. F Allergy Clin Immunol 1999;103:595-600.

6 O'Connor BJ, Ridge SM, Barnes PJ, et al. Greater effect of inhaled budesonide on adenosine-5-monophophate induced than on sodium metabisulphate induced bronchoconstriction in asthma. Am Rev Respir Dis 1992; 146:560-4.

7 Kerrebijn KF, van Essen-Zanduliet ETM, Neijems HJ. Effect of long-term treatment with inhaled corticosteroids and beta-agonists on the bronchial hyperresponsiveness in children. f Allergy Clin Immunol 1987;79:653-9.

8 Haahtela T, Jarvinen M, Kava T, et al. Comparison of a beta-2-agonist, terbutaline, with an inhaled corticosteroid budesonide, in newly detected asthma. N Engl f Med 1991;325:38892.

AUTHORS' REPLY We thank Dr Lipworth for his interest in our article. ${ }^{1}$ Although we found no significant dose difference in $\mathrm{PC}_{20}$ adenosine monophosphate and methacholine or in sputum eosinophils over a two week period between the two doses of fluticasone, the trends suggested a favourable effect of $2000 \mu \mathrm{g}$ compared with $500 \mu \mathrm{g}$ per day for every parameter measured, and there was, indeed, a significant dose response effect on sputum levels of ECP. It is well known that the dose response curve for inhaled steroids in general is very shallow at conventional and higher doses, and we agree that from our data this seems to apply to fluticasone also. From our study, in which only two doses of fluticasone were used, we are careful not to overinterpret where the decline in the therapeutic ratio starts with this drug.

We are aware that the respirable fraction of fluticasone in the dry powder formulation is lower than in the pressurised metered dose inhaler, although the suggested magnitude of the difference is debatable using data from $\mathrm{Dr}$ Lipworth's own group. ${ }^{2}$ Unfortunately, in humans we still have considerable problems in separating the effects of common drugs on the large and the small airways, and the remarks by Dr Lipworth on the site of delivery are intuitively correct but, we believe, unproven as far as the clinical effects are concerned. There is no doubt that the dry powder formulation has systemic bioavailability and we clearly demonstrate this. We accept the notion that, with more sensitive markers of bioavailability, an effect might have been demonstrable also with the dose of $500 \mu \mathrm{g}$ per day. The clinical relevance of this still needs to be determined even after so may years of using inhaled steroids.

We agree that the improvement in hyperresponsiveness with steroid treatment can continue for much longer than the improvement in forced expiratory volume in one second $\left(\mathrm{FEV}_{1}\right) .^{3}$ The concept that the improvement in methacholine hyperresponsiveness might continue for a longer period than that of 
adenosine is interesting, but we are unaware of any data to substantiate this. In fact, in a study by Weersink and colleagues, the same difference between the two bronchoconstrictor agents held true for six weeks instead of the two weeks of fluticasone treatment in the current study.

It is interesting to debate whether the insufficient effect of inhaled steroids in patients with severe asthma is due to lower availability in the peripheral airways, as $\mathrm{Dr}$ Lipworth suggests, or, for instance, to a decreased sensitivity to steroids-either per se or as a result of increased inflammation and associated cytokine load. ${ }^{5}$ The suggestion by Dr Lipworth should result in a relatively better effect of systemic steroids compared with inhaled steroids, especially in the more obstructed patients, but this does not agree with our clinical impression. In fact, the finding of a superior effect of the inhaled corticosteroid over oral prednisolone $(30 \mathrm{mg}$ for two weeks) in our study rather suggests a contrary mechanism, perhaps compatible with a higher effectiveness of the lipophilic compound fluticasone at the level of the epithelium and (sub)mucosa than of systemic prednisolone, even if only in the larger airways. Nevertheless, we are careful not to extrapolate our findings beyond the devices and population studied. There are, however, in addition to ours, a few other studies which suggest that inhaled corticosteroids may have an effect at least as great as prednisolone in asthma exacerbations. ${ }^{6}$

RONALD J MEIJER HUIB A M KERSTJENS DIRKJE S POSTMA Department of Pulmonary Diseases, University Hospital Groningen Groningen, The Netherlands

1 Meijer RJ, Kerstjens HA, Arends LR, et al. Effects of inhaled fluticasone and oral prednisolone on clinical and inflammatory parameters in patients with asthma. Thorax

2 Wilson AM, Sims EJ, Orr LC, et al. Differences in lung bioavailability between different propellants for fluticasone propionate. Lancet 1999 354:1357-8.

3 Kerstjens HAM, Brand PLP, Hughes MD, et al. A comparison of bronchodilator therapy with or without inhaled corticosteroid therapy in obstructive airways disease. $N$ Engl $\mathcal{F}$ Med 1992;327:1413-9.

4 Weersink EJ, Douma RR, Postma DS, et al. Fluticasone propionate, salmeterol xinafoate, and their combination in the treatment of nocturnal their combination in the treatment of nocturnal asthma.

5 Ghaffar O, Hamid Q, Renzi PM, et al. Constitutive and cytokine-stimulated expression of eotaxin by human airway smooth muscle cells. Am f Respir Crit Care Med 1999;159:1933-42.

6 Levy ML, Stevenson C, Maslen T. Comparison of short courses of oral prednisolone and fluticasone propionate in the treatment of adults with acute exacerbations of asthma in primary care. Thorax 1996;51:1087-92.

7 DiFranco A, Giannini D, Bartoli ML, et al. Anti-inflammatory effect of prednisone vs. fluticasone propionate in the treatment of moderate exacerbations of asthma as assessed by sputum analysis. Am f Respir Crit Care Med 1998; 157:A872.

\section{"Systematic review" of asthma education studies}

We were disappointed that Sudre et al felt there was insufficient documentation and excessive variability in studies of education programmes for adults with asthma published between 1979 and 1998. We feel that their conclusion is largely because they did not perform a rigorous systematic review of papers in this area.

Systematic reviews of research evidence are undoubtedly invaluable scientific activities. They establish whether scientific findings are consistent and can be generalised across populations, settings, and other variations. Systematic reviews should be based on the "gold standard" of published randomised clinical trials. However, in the 77 trials reported Sudre et al included 35 studies which were not randomised controlled trials. They also give no information about which interventions were found to have statistically significant effects. They include a study which simply asked patients whether they preferred audiovisual information or written information and did not have any intervention, ${ }^{2}$ a study which has not been published, ${ }^{3}$ and interventions assessing the use of psychotherapy ${ }^{4}$ and yoga ${ }^{5}$ for asthma patients, which seem outside the criteria for inclusion in the review. Another four studies they include are excluded from the Cochrane reviews of patient education ${ }^{67}$ on the grounds that they are not educational intervention studies. It is therefore not surprising that in $81 \%$ of projects assessed the background educational theory was not mentioned and few projects had a patient's needs assessment performed.

While we accept that many of the studies reviewed had missing information on the form and duration of education, we are concerned that some of these studies may be being misquoted. As an example, our own randomised controlled trial on personalised patient education for asthma delivered in four booklets over three months (reference 65) is incorrectly quoted as consisting of "a 10 minute encounter with a physician". ${ }^{8}$ We are concerned that other studies referenced may also have been incorrectly classified.

$$
\begin{array}{r}
\text { GRAHAM DOUGLAS } \\
\text { LIESL OSMAN } \\
\text { Chest Clinic, } \\
\text { Aberdeen Royal Infirmary, } \\
\text { Foresterhill, } \\
\text { Aberdeen } \\
\text { AB25 2ZN, }
\end{array}
$$

$U K$

1 Sudre P, Jacquemet S, Uldry C, et al. Objectives, methods and content of patient education programmes for adults with asthma: systematic review of studies published between 1979 and review of studies published be

2 Partridge MR. Asthma education: more reading Partridge MR. Asthma education: more reading
or more viewing? $\mathcal{F}$ S Soc Med 1986;79:326-8. 3 Simonian YH. The efficacy of education and Simonian YH. The efficacy of education and
resilience training on asthma patients' self resilience training on asthma patients' self
management and quality of life. University of Utah, 1999 .

4 Deter HC. Cost-benefit analysis of psychosomatic therapy in asthma. I Psychosom Res 1986; 30:173-82

5 Nagendra HR, Nagarathna R. An integrated approach of yoga therapy for bronchial asthma: a 3-54 month prospective study. I Asthma 1986;23:123-37.

6 Gibson PG, Coughlan J, Wilson AJ, et al. The effects of limited (information only) patient education programs on the health outcomes of adults with Library, 1997.

7 Gibson PG, Coughlan J, Abramson M, et al. The effects of self-management education and regular practitioner revieww in adults with asthma. Oxford: The Cochrane Library, 1998.

8 Osman LM, Abdalla MI, Beattie JAG, et al. Reducing hospital admission through computer supported education for asthma patients. Grampian Asthma Study of Integrated Care (GRASSIC). BMF 1994;308:568-71.

AUTHORS' REPLY Drs Douglas and Osman correctly state that we did not perform a systematic review of the efficacy of education programmes, but neither did we claim to do so. Our goal, clearly stated in the title, was to describe the objectives, methods, and content of education programmes. In fact, we renounced conducting a meta-analysis of the effectiveness of programmes when we realised the extent of the variability of educational interventions. Averaging the proverbial apples and oranges did not make much sense. Our study suggests that, not only is the number of fruit species greater than anticipated (variability between programmes), but you cannot always tell one from the other (insufficient description of programmes). The latter finding implies that even a systematic review aimed at identifying features associated with greater effectiveness is not feasible. Such an endeavour would be further complicated by the fact that variables used to assess efficacy vary from one evaluation study to the next. In our opinion, standardisation of both programme descriptions and evaluation methods would foster progress in patient education.

While randomised controlled trials are the gold standard for assessing efficacy, all studies reporting an educational intervention should describe in sufficient detail what that intervention consisted of. We therefore included in our review all studies that had an educational component, regardless of the evaluation design.

We admit that we used a broad definition of education as "any attempt to provide the patient with knowledge or personal skills to reduce the impact of asthma on health". The educational content varied among programmes (this is one of our main points) and could include drug management, environmental control, relaxation, yoga, etc. The paper by Partridge ${ }^{1}$ provides an interesting description of an education programme in an asthma clinic, its weaknesses, and attempts at correcting these. As for including work published only as a dissertation, this may be considered an advantage rather than a drawback by some meta-analysts. We maintain that all studies that we reviewed included an explicit educational component and doubt that changing eligibility criteria to exclude a small subset of studies would much alter our general conclusions.

We stand corrected about the incomplete reference to the Grassic intervention in the discussion section of our paper. ${ }^{2}$ In our database this programme was described more accurately as follows (partial data): number of training sessions: 4 (counting one 10 minute session in person and three mailed booklets); duration of training period: 3 months; delivery of education by: physician and self-help; educational setting: individual; training tools: booklet; training method: lecture/vertical teaching. Had we conducted an effectiveness review we would have no doubt singled out this study as by far the largest trial of asthma education, and one that did achieve clinical benefits for its patients. More such research studies are needed.

$$
\begin{array}{r}
\text { T PERNEGER } \\
\text { P SUDRE } \\
\text { C ULDRY } \\
\text { S JACQUEMET } \\
\text { Hôpital Universitaire de Genève, } \\
\text { Rue Micheli du Crest, } \\
1211 \text { Genève, } \\
\text { Switzerland }
\end{array}
$$

1 Partridge MR. Asthma education: more reading or more viewing? $\mathcal{F} R$ Soc Med 1986;79:

2 Osman LM, Abdalla MI, Beattie JAG, et al. Reducing hospital admission through computer supported education for asthma patients. Grampian Asthma Study of Integrated Care
(GRASSIC). BMF 1994;308:568-71. 


\section{BOOK REVIEWS}

Case Presentations in Clinical

Tuberculosis Davies PDO, Ormerod LP. (Pp 320; £29.99). Edward Arnold. ISBN 0 340741597.

This text is a thorough but concise overview of clinical tuberculosis presented as a well structured series of cases with clearly reproduced radiographs, computed tomographic scans, and slides. Each case is complemented by a short pertinent discussion clarifying any points of interest or debate. A carefully chosen chapter layout sequentially introduces the reader to the most challenging and interesting aspects of the disease and also provides an easy reference framework.

The authors' obvious wealth of experience allows readers with a more limited exposure to learn something of the more unusual manifestations of infection, including an extensive range of extrapulmonary and multisystem disease. The complex matter of antituberculous treatment in the emergent group with drug resistant mycobacterial infection, comorbidity, or compliance problems is tackled in some depth, highlighting potential pitfalls and explaining, in a real clinical context, the reasons behind the decisions made.

The difficulties associated with the diagnosis and management of tuberculosis in patients with human immunodeficiency virus are well illustrated, but not exhaustively covered, in a chapter whose commentary sections are particularly full and instructive.

Most of the 120 featured case presentations have a short list of affiliated references aimed to guide, rather than delineate in detail, further research of the points of interest raised.

The format of the book ensures an enjoyable and pragmatic approach to learning about tuberculosis, thus making it directly relevant to all those involved in the medical care of patients with the condition, especially at a training level. It would be an ideal accompaniment to existing formal textbooks.-ILJ

Anti-Inflammatory Drugs in Asthma. Sampson AP, Church MK, eds. (Pp 288, hardback). Switzerland: Birkhauser, 1999. ISBN 3764358734

This is one of a series of publications under the collective heading "Progress in Inflammation Research" to which some of the European heavyweights in asthma research have contributed chapters. All the asthma drugs are included with the notable exception of the anticholinergic agents, although I found the title a little misleading as the in vivo anti-inflammatory effects of some of the drugs discussed remains contentious. However, from the opening chapter it becomes apparent that investigations into the pathophysiology of, and the effects of treatment on, asthma have played an important part in defining the inflammatory mechanisms. The "commonly" used asthma medications are discussed initially with Peter Barnes giving an erudite synopsis of the anti-inflammatory effects of corticosteroids. The next two chapters deal with the putative anti-inflammatory effects of phosphodiesterase inhibitors and $\beta_{2}$ adrenoceptor agonists, although the chapter on phosphodiesterase inhibitors concentrated on the different isoenzymes and thus was heavy going with little discussion of their anti-inflammatory effects and no concluding summary. Despite theophylline being available for at least 40 years, I was struck by the paucity of clinical data available regarding its efficacy and in vivo anti-inflammatory effect (if at all). This is presumably because it is not profitable for pharmaceutical companies to investigate the drug further. The mast cell stabilisers are considered next, and the last third of the book deals with leukotriene antagonists and discusses other novel potential anti-inflammatory agents including antiIgE agents, cytokines and adhesion molecule antagonists.

Several of the chapters are interesting and well written with well laid out tables and graphs, although some have several annoying typographical errors. The book does provide a good summary of the anti-inflammatory effects of present and potential future asthma medications and would act as a good reference source for departments or individuals with an interest in this field. - JB

\section{NOTICES}

\section{Cardiovascular Disease Prevention V}

A conference entitled "Cardiovascular Disease Prevention V" will be held on 4-7 April 2000 at the Conference Centre, Kensington Town Hall, London. For further information contact The Secretariat, Hampton Medical Conferences Ltd, 127 High Street, Teddington, Middlesex TW11 8HH, UK. Telephone +44 (0) 1819770011 . Fax +44 (0)1819770055. email hmc@hamptonmedical.com

\section{British Association for Lung Research}

The British Association for Lung Research (BALR) Spring Meeting entitled "Inflammation Control: A Goal for the Millenium" will be held on 18 April 2000 at the Wills Hall, University of Bristol. For further information contact Dr Lynne Armstrong, The Lung Research Group, University of Bristol Medical School Unit, Southmead Hospital, Westbury on Trym, Bristol BS10 5NB, UK. Telephone +44 (0)117959 5348. Fax +44 (0)117959 5018. email Lynne.Armstrong@bristol.ac.uk

\section{CORRECTIONS}

\section{UK Pulmonary Vascular Units}

In the list of UK Pulmonary Vascular Units given at the end of the review article on "Primary pulmonary hypertension" by A J Peacock which appeared in the December issue of Thorax (1999;54:1107-18), the address for Dr Simon Gibbs should have included the Imperial College School of Medicine which includes Hammersmith, Brompton and Harefield hospitals.

\section{Atrial septostomy in pulmonary vascular disease}

In the editorial entitled "Role of atrial septostomy in the treatment of pulmonary vascular disease" by R J Barst which appeared on pp 95-6 of the February issue of Thorax, there was an error in figure 1 . The correct version is reproduced below, showing that in "non-responders" the PAP is increased or unchanged. The publishers apologise for this error.

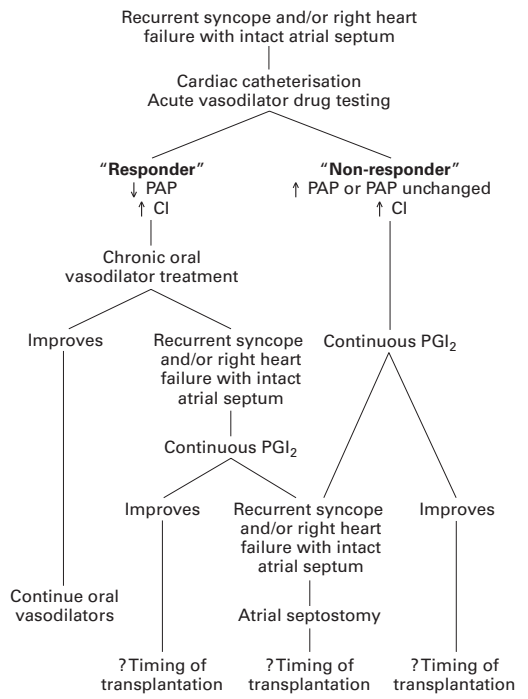

